\title{
Analysis of the supportive care needs of the parents of preterm children in South Korea using big data text-mining: Topic modeling
}

\author{
Ji Hyeon Park ${ }^{1}$, Hanna Lee ${ }^{1}$, Haeryun Cho ${ }^{2}$ \\ ${ }^{1}$ Graduate Student, Department of Nursing, Wonkwang University, Iksan; ${ }^{2}$ Associate Professor, Department of Nursing, Wonkwang University, Iksan, Korea
}

Purpose: The purpose of this study was to identify the supportive care needs of parents of preterm children in South Korea using text data from a portal site. Methods: In total, 628 online newspaper articles and 1,966 social network service posts published between January 1 and December 31, 2019 were analyzed. The procedures in this study were conducted in the following order: keyword selection, data collection, morpheme analysis, keyword analysis, and topic modeling. Results: The term "yirundung-yi", which is a native Korean word referring to premature infants, was confirmed to be a useful term for parents. The following four topics were identified as the supportive care needs of parents of preterm children: 1) a vague fear of caring for a baby upon imminent neonatal intensive care unit discharge, 2) real-world difficulties encountered while caring for preterm children, 3) concerns about growth and development problems, and 4) anxiety about possible complications. Conclusion: Supportive care interventions for parents of preterm children should include general parenting methods for babies. A team composed of multidisciplinary experts must support the individual growth and development of preterm children and manage the complications of prematurity using highly accessible media.

Key words: Big data; Child; Parents; Preterm infant; Data mining

\author{
Corresponding author Haeryun Cho \\ https://orcid.org/0000-0001-7366-9774 \\ Department of Nursing, Wonkwang University, \\ 460 Iksn-daero, Iksan 54538, Korea \\ TEL +82-63-850-6020 FAX +82-63-850-6060 \\ E-MAIL chr@wku.ac.k \\ *This study was supported by a National Research Foundation of Korea (NRF) \\ grant funded by the Korean government (No. NRF-2019R1I1A3A01060632).
}

Received Oct 30, 2020 Revised Dec 11, 2020 Accepted Dec 21, 2020

(a) This is an Open Access article distributed under the terms of the Creative Commons Attribution NonCommercial License (http://creativecommons.org/licenses/by-nc/4.0/) which permits unrestricted noncommercial use, distribution, and reproduction in any medium, provided the original work is properly cited.

\section{INTRODUCTION}

Worldwide, the proportion of premature births (under 37 weeks of gestation) is approximately $10 \%$ [1]. In South Korea, the proportion of premature infants has more than doubled from $3.8 \%$ in 2000 to $8.1 \%$ in 2019 [2]. Meanwhile, the survival rate of premature infants is steadily increasing with advances in medical technology and the implementation of active policies related to the neonatal intensive care unit (NICU) [3]. According to University of Utah Health, the survival rate for infants born at 24 weeks is approximately $50 \%$, but $80 \%-90 \%$ for infants born at 28 weeks and $95 \%$ at 32 weeks [4]. In South Korea in 2019, the survival rate was $87.9 \%$ for infants born with a body weight under $1,500 \mathrm{~g}$ and $72.8 \%$ for those under $1,000 \mathrm{~g}$ [2].

As the survival rate of premature infants improves, the interest in the field has shifted from medical care to parenting at home after NICU discharge and the proper growth and development of premature children [3,5]. Even if premature infants are safely discharged, they are at a high risk of complications, such as health problems or developmental disabilities [6,7].

Parents of infants born prematurely are exposed to various crisis situations while coping with the possible health problems and developmental disabilities of their children [8]. They 
also experience more and longer post-traumatic and parenting stress than the parents of full-term infants $[9,10]$ and encounter anxiety, depression, and conflict $[8,11]$. They may be unprepared for their parental roles or may exhibit inappropriate parenting behaviors, such as overprotection $[11,12]$.

Parental responses are the major factor influencing the neurodevelopment, cognitive development, and behavioral development of children born as premature infants [13]. Parents experiencing high parenting stress have impaired mental health, inappropriate relationships with their children, and poor parenting quality $[5,14]$. Therefore, the need for interventions that can support the parents of preterm children has emerged [5,8,12].

The term "big data" refers to the vast amount of data generated online, which would be difficult to analyze using existing data processing technologies [15]. Currently, online social network services (SNSs) are used for communication by a large number of people, and they can be used to grasp various viewpoints comprehensively because of the amount of data that is accumulated [16]. In particular, South Korea has the highest internet penetration rate in the world, and more than $50 \%$ of newspaper users use online platforms $[15,16]$. Big data analysis using text data of online news and SNSs has been attracting attention as a useful analytical method to explore the flow of people's perception, times, and society $[15,16]$. Topic modeling is an analytical method that can find hidden topics in a large amount of text data [15]. Moreover, research using surveys and interviews is limited in terms of identifying population-scale phenomena owing to the selection of participants. By contrast, the analysis of big data, including online newspapers and SNSs, can reflect the experiences of the population [15].

Interest in the growth, development, and parenting of preterm children has been increasing. Related research is being conducted worldwide; some examples include a meta-analysis of the parenting behavior of parents of preterm children [12], qualitative studies on the care experience of preterm children $[8,11]$, a systematic literature review of the relationship between parenting style and preterm infant development [13], and research on the stress of parenting preterm children $[5,9,17]$. These studies have reported that support is required for the parents of preterm children, but have been limited to investigations of the relationships between parenting aspects and child development. In other words, research aiming to understand the types of support required by the parents of preterm children is insufficient.

Research on the experiences of parents of preterm children after their discharge from the NICU is insufficient owing to the limited accessibility to such parents. However, analyzing the experiences of parents of preterm children using online big data is expected to produce results that reflect the actual phenomena because there are no restrictions on participation. Online news can be regarded as a public concern because it contains information that attracts attention related to real life through interactions with people living at the same time [18]. Therefore, this study was conducted to identify the types of support requests made by the parents of preterm children by using text data from online newspaper articles and SNS posts on a portal site. The specific purpose of the present study was 1) to collect big data using text mining to analyze keywords and 2) to derive the supportive care needs of parents of preterm children by using topic modeling.

\section{METHODS}

\section{Study Design}

In this study, text mining was performed using online big data to identify the supportive care needs of parents of preterm children.

\section{Sample}

The portal site used was Naver, which has been reported to be the most widely used search engine in South Korea, with a market share of more than $60 \%[19]$. The researchers conducted face-to-face meetings six times to closely examine agreement for the selection of texts to be used in the analysis of the supportive care needs of parents of preterm children using big data text mining. In total, 1,247 online newspaper articles and 1,966 SNS posts by the parents of preterm children in a web community on a portal site, posted between January 1 and December 31, 2019, were analyzed.

\section{Study Process and Data Analysis}

This study was conducted in the following order: keyword selection, data collection, morpheme analysis, keyword analysis, and topic modeling (Figure 1).

\section{1) Keyword selection}

The keywords selected were "premature infant" or "yirundung-yi". Officially, the term "premature infant" is used, but the National Institute of the Korean Language created a new term, "yirundung-yi", in 2006. "Yirundung-yi" is a native Korean word that denotes that a baby was born earlier than full term [20], and it is commonly used in online newspaper articles and SNSs.

\section{2) Data collection}

Data were collected through Textom (Textom, the IMC, 


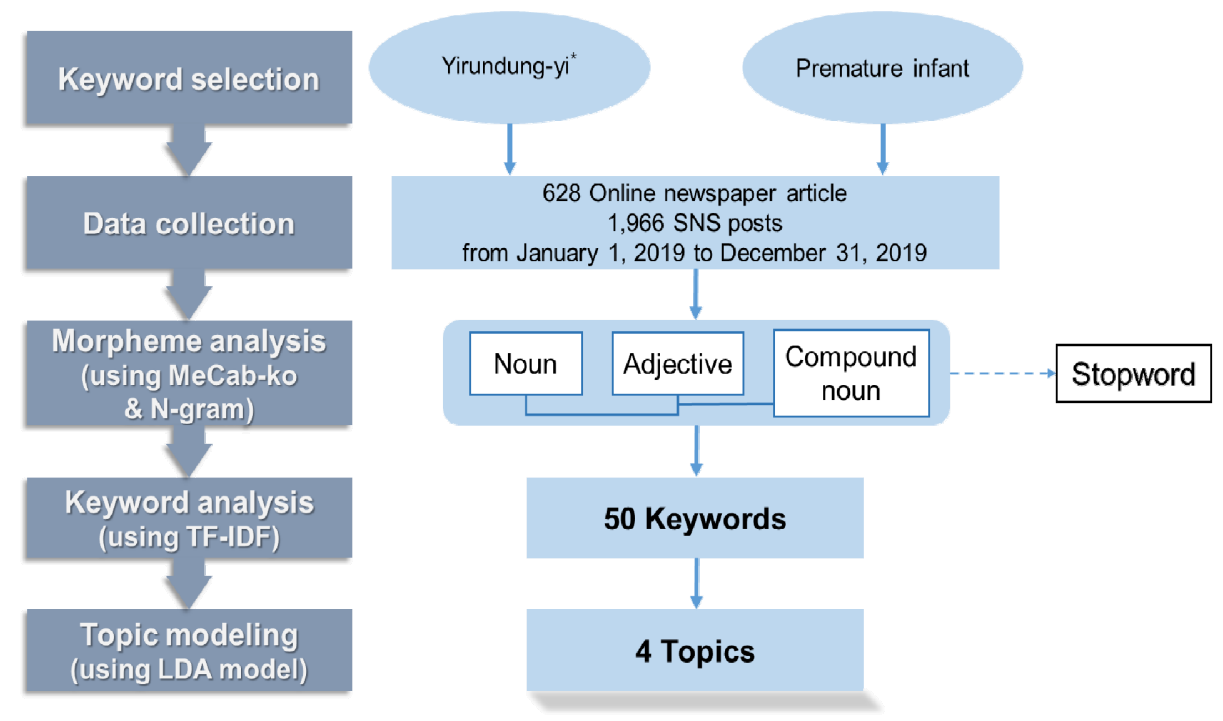

Figure 1. Study process and data analysis. *Yirundung-yi is native Korean as premature infant; LDA, latent dirichlet allocation; SNS, social network service; TF-IDF, term frequency inverse document frequency.

South Korea), a big data analysis software that can collect, clean, and process matrix data in a web environment. It was chosen for this study because it can collect and analyze data from various channels, including portal sites $[15,16]$. The settings for the data collection were as follows: 1) text in Korean; 2) keywords of "yirundung-yi" and "premature infant"; 3) full text; 4) post start date was January 1, 2019; 5) post end date was December 31,$2019 ; 6$ ) the collection channel was an online newspaper article and web community on Naver; and 7) duplicate uniform resource locators (URLs) were removed.

The title, URL, and text of the collected data were saved as an Excel file with a size of 1.2 MB. The titles and texts of the collected data were checked by the researchers, and unrelated data and duplicate content were deleted. In total, 1,247 online newspaper articles were collected, from which 628 texts were analyzed after excluding 583 duplicate and 36 unrelated articles. In total, 1,976 SNS posts were collected; there were 10 unrelated posts and no duplicate data, resulting in 1,966 texts for analysis. The final text size was $627 \mathrm{~KB}$.

\section{3) Morpheme analysis}

Morphological analysis was performed with nouns and adjectives using the MeCab-ko analyzer. Unlike Espresso K, MeCab-ko has the advantage of classifying vocabulary by referring to a dictionary regardless of space [21]. However, as compound nouns are extracted as simple nouns, and synonyms and similar words cannot be distinguished, the meaning of the text was not clear. Thus, through an N-gram depicting the degree of concentration of the keywords, complex nouns were created again from the refined data. Subsequently, a user dictionary was used to refine words with the same or similar meaning and words that did not have a meaning in the refined data. The user dictionary refers to a function in which the words to be changed are constructed as a dictionary when it is necessary to refine data for similar or identical subjects repeatedly, and these words are collectively changed into corrected words designated in the morpheme analysis process [21]. Therefore, a synonym dictionary was constructed for words with agreed-upon meanings, and words with similar meanings were constructed as a thesaurus dictionary using Textom's user word dictionary. Words such as "because of", "etc.", "related", and "and" do not have relevant meanings in this context; therefore, they were treated as stop words. The final refined data were saved as a .txt file, and the encoding was set to UTF-8, saved, and then uploaded to Textom.

\section{4) Keyword analysis}

In this study, the term frequency-inverse document frequency (TF-IDF) value was applied to extract important keywords, as it represents the frequency and weight of specific words in the document [22]. From the collected data, the top 50 keywords were extracted by applying the TF-IDF weight to each data point. In addition, the keywords were analyzed with the keywords of data collection: "yirundung-yi" and "premature infant". A word cloud is the most representative text mining technique for visualizing keywords [23]. In this study, the size was expressed as large, medium, and small according to the frequency of a word's appearance in the data. The colors were set as blue, green, and gray, in descending order of importance of appearance in the data. 


\section{5) Topic analysis}

For topic modeling, the latent dirichlet allocation (LDA) model was used in this study. The LDA method assumes that a document is a collection of topics, each of which is a set of keywords, and it probabilistically infers the topics latent in the document [24]. In the LDA method, the researcher determines the number of topics that are considered well-categorized [21]. In this study, the number of topics was determined to be four, which demonstrated the clearest distance and boundary between the topics. The researchers reviewed the keywords and texts that composed the four topics that were derived and named them in a way that clearly expressed their meaning.

\section{RESULTS}

\section{Keyword Analysis}

The top 50 keywords based on their TF-IDF values are shown in Table 1. Three word clouds for the combined data (online newspaper articles and SNS data), online newspaper article data, and SNS data are presented in Figure 2. The word cloud of online newspaper articles revealed that "premature infant" was the most important term, as its representation in the cloud was larger than that of "yirundung-yi", indicating that the official medical term was primarily used in online newspaper articles. In the word cloud of SNS data, "yirundung-yi" was found to have higher importance than "premature infant", and other words related to parenting were found to be the main words.

\section{Topic Modeling}

The four topics using LDA modeling are shown in Figure 2 and discussed in the following sections.

\section{1) Vague fear of caring for a baby from the time when NICU dis- charge is imminent}

The main keywords for the first topic were "premature infant formula", "discharge", and "incubator". This topic indicates that the parents started preparing for their parental role when their infants neared their discharge from the NICU, and that they experienced vague anxiety.

I'm a yirundung-yi mom, but I'm still very unfamiliar. I don't know anything. What is corrected age? (code 1920)

I am a yirundung-yi mom of a 37-week and 1,750 g baby. The baby is still in the incubator, and it is about to be three weeks of hospitalization. I think he/she will be discharged this month. What are the hospital expenses and supplies for discharge? (code 1165)

\section{2) Real-world difficulties encountered while caring for preterm children}

The main keywords that composed the second topic were "Naturemade?", "infant formula", and "recommendation". This topic encompassed the real-world problems that the parents faced directly after their infants were discharged from the NICU. Unlike the first topic, it dealt with the specific and real-world difficulties of child-rearing, such as feeding and providing clothes and diapers.

My baby was discharged from the NICU today and We couldn't find a place to buy premature infant formula. Does anyone know where premature infant formula is sold, or someone who wants to sell leftover premature infant formula? (code 319)

My baby was finally discharged yesterday and came home. Whether it is day or night, the baby keeps shouting and moving. The baby constantly has strong movements throughout the whole body, is it okay? (code 366)

It is a reality that special diapers and powdered milk that are needed for yirundung-yi are difficult to obtain at home because they are not only expensive but also in limited supply. The Ministry of Health and Welfare, Samsung Card, and the Green Umbrella Children's Foundation support the 'childcare kit.' (code 2027)

\section{3) Concerns about the growth and developmental problems of preterm children}

The third topic was composed of "worry", "weight", "test", "retinopathy of prematurity (ROP) test", and "corrected age". This topic indicates that after discharge from the NICU, parents became aware of the problems related to the growth and development of their children and became anxious or concerned.

My baby was born as yirundung-yi, and corrected age is seven months. The counselor doctor in the portal site and the attending physician said that the yirundung-yi's development will be 2-3 months later than full-term children. My baby is 7 months old, but he still doesn't try to put weight on his legs. (code 1524)

I am a beginner mom raising a 46-day-old baby. The baby was born at 36 weeks, and the weight and height were similar to those of other children, but he was born a month early, so it seemed that he never opens his eyes and plays. He opened his eyes for a very short time and cried, and after I hugged him, he fell asleep again. It seems that only my baby is late. (code 437) 
Table 1. Top 50 Keywords $(N=9,782)$

\begin{tabular}{|c|c|c|c|c|c|c|}
\hline \multirow{2}{*}{ Rank } & \multicolumn{2}{|l|}{ Total $(N=9,782)$} & \multicolumn{2}{|c|}{ Online newspaper articles $(\mathrm{n}=5,451)$} & \multicolumn{2}{|l|}{ SNS $(n=5,304)$} \\
\hline & Keyword & TF-IDF & Keyword & TF-IDF & Keyword & TF-IDF \\
\hline 1 & Yirundung-yi & $2,132.5$ & Yirundung-yi & 824.2 & Yirundung-yi & $1,262.5$ \\
\hline 2 & Premature infant & $1,660.2$ & Premature infant & 626.0 & Premature infant & 906.8 \\
\hline 3 & Premature infant formula & $1,041.0$ & Newborn & 416.8 & Premature infant formula & 849.7 \\
\hline 4 & Infant & 988.9 & Medipost Pneumostem ${ }^{\circledR} *$ & 404.3 & Naturemade $^{\circledR \dagger}$ & 810.1 \\
\hline 5 & Naturemade $^{\circledR \dagger}$ & 987.0 & Daughter & 360.5 & Infant & 714.8 \\
\hline 6 & Child & 817.2 & Under & 336.5 & Powdered milk & 574.3 \\
\hline 7 & Newborn & 722.6 & NICU & 324.5 & Give & 505.5 \\
\hline 8 & Powdered milk & 706.4 & Child & 322.4 & Baby & 504.6 \\
\hline 9 & Hospital & 646.3 & Born & 306.8 & Discharge & 497.1 \\
\hline 10 & Discharge & 631.0 & Pregnancy & 298.3 & Child & 494.3 \\
\hline 11 & Baby & 611.0 & Incubator & 289.0 & Yirundung-yi mom & 474.1 \\
\hline 12 & Give & 609.3 & ROP & 277.2 & Hospital & 402.5 \\
\hline 13 & Huggies $^{\circledR}$ & 601.3 & Support & 275.9 & Weight & 361.6 \\
\hline 14 & Incubator & 584.2 & BPD & 257.4 & Huggies $^{\circledR}$ & 348.7 \\
\hline 15 & Daughter & 574.6 & Particulate matter & 254.5 & Need & 323.8 \\
\hline 16 & Yirundung-yi mom & 564.1 & Huggies $^{\circledR}$ & 253.5 & Delivery & 322.8 \\
\hline 17 & Childbirth & 533.5 & Congenital anomaly & 252.8 & Breast milk & 314.3 \\
\hline 18 & Medipost Pneumostem ${ }^{\circledR *}$ & 524.7 & Hospital & 243.9 & Degree & 310.1 \\
\hline 19 & Under & 515.7 & Danger & 241.9 & Babe & 305.8 \\
\hline 20 & Weight & 505.3 & Voucher & 232.9 & Sale & 298.7 \\
\hline 21 & NICU & 500.9 & Infant & 224.4 & Postnatal care center & 286.4 \\
\hline 22 & Birth & 488.4 & Treatment & 221.5 & Mother & 286.1 \\
\hline 23 & Need & 478.0 & Pregnant woman & 219.2 & Height & 280.8 \\
\hline 24 & Pregnancy & 466.7 & Person & 216.3 & Preemie & 280.0 \\
\hline 25 & Mother & 464.0 & Delivery & 210.8 & Incubator & 274,4 \\
\hline 26 & Support & 454.7 & Body weight & 205.3 & Recommendation & 270.6 \\
\hline 27 & Breast milk & 397.6 & Diaper & 202.2 & Nicu & 266.9 \\
\hline 28 & Degree & 377.8 & Yuhan-Kimberly & 202.2 & ROP & 261.5 \\
\hline 29 & Body weight & 364.4 & Selection & 195.0 & Worry & 258.0 \\
\hline 30 & $\mathrm{ROP}$ & 364.4 & Health & 193.0 & Birth & 253.5 \\
\hline 31 & Congenital anomaly & 361.8 & Region & 188.4 & Completion & 252.7 \\
\hline 32 & Sale & 358.1 & Medical expenses support & 183.4 & Test & 250.3 \\
\hline 33 & Delivery & 356.0 & Campaign & 181.8 & Mom & 248.9 \\
\hline 34 & Health & 354.9 & Extreme prematurity & 181.8 & Corrected & 235.1 \\
\hline 35 & Babe & 352.0 & Mother & 177.9 & Twin & 231.3 \\
\hline 36 & Twin & 350.5 & Parent & 175.2 & Thinking & 228.4 \\
\hline 37 & Particulate matter & 339.3 & Hospital bills & 173.2 & Infant formula & 215.5 \\
\hline 38 & Height & 338.6 & Hold & 170.6 & University hospital & 212.4 \\
\hline 39 & Treatment & 337.2 & Surgery & 168.4 & Newborn & 210.4 \\
\hline 40 & $\mathrm{BPD}$ & 334.1 & Ministry of Health and Welfare & 168.1 & Small & 205.2 \\
\hline 41 & Danger & 330.3 & Outbreak & 167.4 & Request & 203.4 \\
\hline 42 & Postnatal care center & 329.0 & Doctor & 166.6 & Home & 202.4 \\
\hline 43 & Premature retina & 323.8 & World & 159.0 & Hospitalization & 201.3 \\
\hline 44 & Surgery & 323.0 & Puerperal mother & 158.7 & ROP test & 196.2 \\
\hline 45 & Preemie & 318.6 & Child Fund Korea & 156.3 & Collect on delivery & 192.5 \\
\hline 46 & Person & 315.9 & Case & 155.3 & Use & 190.9 \\
\hline 47 & Worry & 311.6 & This year & 153.0 & Agonize & 188.3 \\
\hline 48 & Recommendation & 308.2 & Need & 151.9 & Post & 180.9 \\
\hline 49 & Voucher & 306.9 & The latest & 149.8 & Premature birth & 179.2 \\
\hline 50 & Nicu & 306.9 & Last year & 147.2 & Corrected age & 171.4 \\
\hline
\end{tabular}

${ }^{*}$ A medication for preventing and treating bronchopulmonary dysplasia in clinical trials in South Korea; ${ }^{\dagger}$ A diaper brand name in South Korea; BPD, bronchopulmonary dysplasia; NICU, neonatal intensive care unit; ROP, retinopathy of prematurity; SNS, social networking service; TF-IDF, term frequency-inverse document frequency. 
(A)

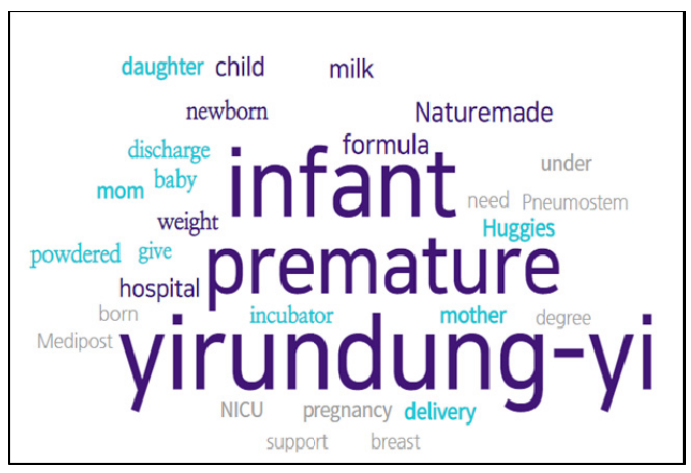

(B)

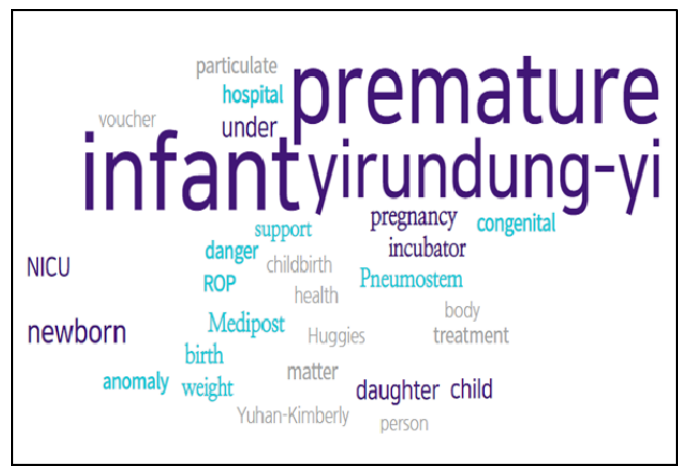

(C)

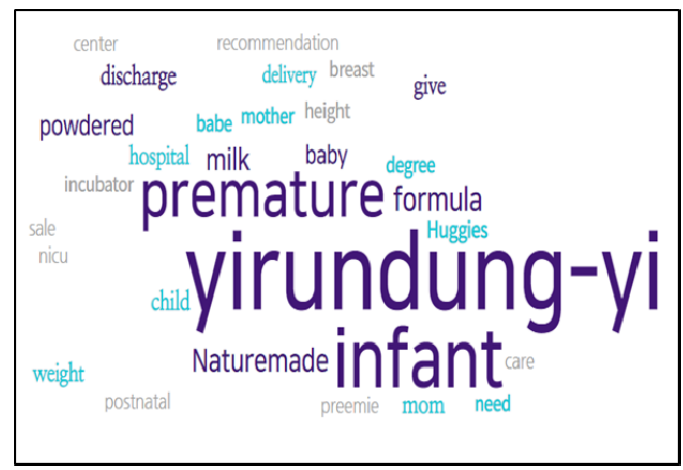

Figure 2. Word clouds. (A) Total. (B) News articles. (C) Social network service.

Everyone around me tells me not to worry too much because the kids will grow up on their own when the time comes. But when my child seems to be developing or growing later than his peers, I inevitably become anxious. When should my baby have an infant and toddler development test? (code 2445)

\section{4) Anxiety about possible complications in preterm children}

The main keywords that composed the fourth topic were "retinopathy of prematurity (ROP)", "bronchopulmonary dysplasia", "treatment", "medical expenses support", and "surgery". This topic indicated worries about potential future health problems, such as future complications and treatment prognosis.

My baby was hospitalized for 5 days with jaundice and had a level of 19.5 at 4 days old. He was 6 months and 9 days old. Should he be going for a retinopathy of prematurity test? (code 365)

My baby was born as a very premature infant, and his corrected age is 6 months. During the weekend, he had phlegm and coughed quite a bit. We went to a nearby pediatrician in a hurry to get a prescription, but the he didn't have any lung sickness yet. When he wheezes, it is called bronchiolitis. What should I do? (code 720)

Medipost Pneumostem ${ }^{\circledR}$ is a treatment for bronchopulmonary dysplasia, which is mainly composed of mesenchymal stem cells derived from allogeneic cord blood. Bronchial pulmonary dysplasia is a chronic lung disease that occurs in premature infants receiving artificial ventilation and oxygen therapy. (code 2451)

\section{DISCUSSION}

This study analyzed a vast amount of text from online newspaper articles and SNS posts to understand the supportive care needs reported by parents raising preterm children. Four topics were identified through the data analysis.

The first topic was parents' vague fear of caring for their child, starting when NICU discharge was imminent. First-time parents generally experienced stress and anxiety about caring for their children [5]. In particular, previous studies have reported that the parents of premature infants are generally less prepared for parenting than the parents of full-term infants [11]. Furthermore, the parents of premature infants experience more stress than the parents of full-term infants [9,25]. Parent education [26], skin-to-skin contact through kangaroo care [27], and massages [28] are among the interventions that have 
been implemented in NICUs to enhance parent-child attachment, reduce parental stress, and strengthen parental confidence. However, in this study, it was found that parents exhibited an uncertain and vague reaction to caring for their children. Although NICU discharge education is being implemented, in reality, parents seek advice on how to handle their children using SNS, which indicates that the education provided at the NICU was ineffective. Therefore, it is necessary to develop effective educational methods and content that can improve parents' confidence to raise their children at home after discharge from the NICU.

The second topic encompassed the real-world difficulties of parenting preterm children. Compared to the first topic, which represented an abstract future difficulty, this topic included specific parenting difficulties. According to a study by Lee and Koh [29], the parents of infants lacked the ability to meet their own parenting expectations. In addition, according to a study by Jeong and Kim [30] that analyzed the parental education of parents of infants, the most frequently encountered type of parental education dealt with child development; however, parents wanted education related to actual parenting behaviors, including interacting with their child, solving health problems, and proper eating habits. Consistent with previous studies, the results of this study revealed that keywords related to practical care for infants, such as "formula", "diapers" and "childcare products" appeared, rather than content specific to preterm children. Therefore, the demands of parents who care for their children after discharge from the NICU relate to real-world parenting behaviors. Furthermore, previous studies have reported that similar issues caused stress when parenting premature and full-term infants $[5,10,17]$. In other words, it is necessary to educate the parents of preterm children about general parenting behaviors and to provide interventions that can improve overall parenting competence. In addition, to effectively deliver necessary information to parents caring for infants after being discharged from the NICU, it is necessary to develop educational material that is as accessible as SNSs as a way to find information.

The third topic involved concerns about the growth and development of preterm children. The results of a qualitative study by Kim [8], which analyzed parenting experience with infants born prematurely, indicated that vague anxiety was the parents' main reaction and was caused by a lack of certainty about the normal growth and development of their children. This is consistent with the results of this study. Furthermore, Whittingham et al. [11] also reported that the parents of prematurely born infants lacked assurance that their children would develop appropriately. Parents obtain information on growth and development problems that may occur in premature infants through SNSs or online commun- ities $[5,8,10]$. If parents have a limited ability to interpret information and apply it under the circumstances of caring for a child born as a premature baby, the information related to growth and development provided by health non-professionals may cause excessive anxiety. Therefore, to provide assurance regarding premature children's growth and development, health professionals need to help parents through individual child growth and development interventions, in addition to the use of self-help groups, such as SNSs, by parents of preterm children.

The final identified topic was parental anxiety about possible future health complications in preterm children. Parents of preterm infants are aware of the potential medical problems of their children and seek information [11]. A study by Kim [8] reported that parents of premature infants were anxious to prevent infection, diseases, or complications from worsening after discharge, which is similar to the results of this study. Therefore, it is necessary to provide multidisciplinary interventions for the parents of preterm children, including a neonatal health care professional, a developmental expert, and an expert who can provide medical interventions.

In the word cloud, it was found that the keywords "premature infant" and "yirundung-yi" were similar in size in the online newspaper articles, whereas "yirundung-yi" was larger in the SNS posts where parents' writings were reflected. This result means that for parents, the term "yirundung-yi", which is a pure Korean word, is a more practical term than the technical term "premature infant". Therefore, when providing intervention for the parents of preterm infants, the use of the term "yirundung-yi" rather than the medical term "premature infant" may be a more useful strategy.

As research on raising premature children discharged from the NICU is just beginning, this study is significant in that an analysis was conducted using big data with the goal of understanding the supportive care needs of parents raising preterm children. The significance of this study lies in its ability to identify parents' needs meaningfully because it analyzed 2,594 texts using text mining and LDA topic modeling to extract topics embedded in online documents used by these parents. In addition, as the data were collected using various channels, namely online newspaper articles and SNSs, the research results derived in this study are reliable.

However, a methodological limitation is that the subjectivity of the researcher may have been reflected in the process of refining the keywords. Furthermore, slang, abbreviations, and new words were not completely reflected in the refined keywords. In addition, the results of this study should be interpreted considering the fact that they did not reflect the needs of parents who do not use SNSs. 


\section{CONCLUSION}

The supportive care needs of parents of preterm children were identified in this study and can be summarized as follows: 1) uncertainty in caring for their infants, 2) real-world difficulties in raising their children, 3) concerns about appropriate growth and development, and 4) anxiety about potential medical problems of their children. Reflecting these needs, the following interventions are needed. It is necessary to develop and apply effective education that increases the confidence of parents in caring for their children at home when NICU discharge is imminent. For interventions after NICU discharge, parenting methods specific to premature infants are important; however, information on parenting methods in general should also be included. In addition, it is necessary for a team composed of multidisciplinary experts, such as doctors, nurses, development experts, and occupational therapists, to provide support for the individual growth and development of preterm children and help manage complications of prematurity. Finally, it is suggested that various media such as SNS, internet, and mobile platforms be used to increase the accessibility of information for parents.

\section{Conflict of interest}

No existing or potential conflict of interest relevant to this article was reported.

\section{Data availability}

Please contact the corresponding author for data availability.

\section{REFERENCES}

1. World Health Organization. Preterm birth [Internet]. Geneva: World Health Organization; 2018 [cited 2020 September 10]. Available from:

http://www.who.int/news-room/fact-sheets/detail/preterm-birth

2. Statistics Korea. 2019 Birth statistics [Internet]. Daejeon: Statistics Korea; 2020 [cited 2020 August 28]. Available from:

http://kostat.go.kr/portal/korea/kor_nw/1/2/3/index.board? bmode $=$ read\&bSeq $=\& a S e q=384631 \&$ pageNo $=1 \&$ row $N u m=10 \&$ navCount $=10 \&$ currPg $=\&$ searchInfo $=\&$ Target $=$ title\&sTxt $=$

3. Blencowe H, Cousens PS, Oestergaard MZ, Chou D, Moller AB, Narwal R, et al. National, regional, and worldwide estimates of preterm birth rates in the year 2010 with time trends since 1990 for selected countries: A systematic analysis and implications. Lancet. 2012;379(9832):2162-2172.

https://doi.org/10.1016/S0140-6736(12)60820-4

4. University of Utah Health. Health outcomes for preemies [Inter- net]. Salt Lake City: University of Utah Health; 2020 [cited 2020 September 1]. Available from:

https://healthcare.utah.edu/womenshealth/pregnancy-birth/pre term-birth/when-is-it-safe-to-deliver.php

5. Kim K, Lee H. Parenting stress in preterm and full-term infant mothers by their children's developmental stages. Journal of the Korean Society of Maternal and Child Health. 2020;24(3):162-169. https://doi.org/10.21896/jksmch.2020.24.3.162

6. Purdy IB, Craig JW, Zeanah P. NICU discharge planning and beyond: Recommendations for parent psychosocial support. Journal of Perinatology. 2015;35:S24-S28.

https://doi.org/10.1038/jp.2015.146

7. Gardon L, Piccioloini O, Squarza C, Frigerio A, Gianni ML, Gangi $S$, et al. Neurodevelopmental outcome and adaptive behaviour in extremely low birth weight infants at 2 years of corrected age. Early Human Development. 2019;128:81-85.

https://doi.org/10.1016/j.earlhumdev.2018.12.013

8. Kim JS. Parenting experience of prematurity parents based on grounded theory. Journal of the Korean Society of Maternal and Child Health. 2019;23(4):232-240.

https://doi.org/10.21896/jksmch.2019.23.4.232

9. Suttora C, Spinelli M, Monzani D. From prematurity to parenting stress: The mediating role of perinatal post-traumatic stress disorder. European Journal of Developmental Psychology. 2014;11 (4):478-493. https:// doi.org/10.1080/17405629.2013.859574

10. Howe TH, Sheu CF, Wang TN, Hsu YW. Parenting stress in families with very low birth weight preterm infants in early infancy. Research in Developmental Disabilities. 2014;35(7):1748-1756. https://doi.org/10.1016/j.ridd.2014.02.015

11. Whittingham K, Boyd RN, Sanders MR, Colditz P. Parenting and prematurity: Understanding parent experience and preferences for support. Journal of Child and Family Studies. 2014;23:10501061. https://doi.org/10.1007/s10826-013-9762-x

12. Toscano C, Soares I, Mesman J. Controlling parenting behaviors in parents of children born preterm: A meta-analysis. Journal of Developmental and Behavioral Pediatrics. 2020;41(3):230-241. https://doi.org/10.1097/DBP.00000000000000762

13. Neel MLM, Stark AR, Maitre NL. Parenting style impacts cognitive and behavioural outcomes of former preterm infants: A systematic review. Child: Care, Health and Development. 2018;44(4):507-515. https://doi.org/10.1111/cch.12561

14. Lee SM. Factors influencing parenting stress in mothers of preschoolers born prematurely. Child Health Nursing Research. 2017; 23(4):470-478. https://doi.org/10.4094/chnr.2017.23.4.470

15. Kang J, Kim S, Roh S. A topic modeling analysis for online news article comments on nurses' workplace bullying. Journal of Korean Academy of Nursing. 2019;49(6):736-747. https://doi.org/10.4040/jkan.2019.49.6.736

16. Lee SA, Choi BS, Choi J. Analysis of developmental disabilities news paver articles using Text mining: Focused on early screening 
and early intervention. Journal of Special Children Education. 2020;22(1):1-27. https://doi.org/10.21075/kacsn.2020.22.1.1

17. Gray PH, Edwards DM, O'Callaghan MJ, Cuskelly M. Parenting stress in mothers of preterm infants during early infancy. Early Human Development. 2012;88(1):45-49.

https://doi.org/10.1016/j.earlhumdev.2011.06.014

18. Wang S, An SK. The effect of online news use motivation on acceptance and satisfaction a comparative study on Korean and Chinese university students. Journal of the Korea Contents Association. 2020;20(6):293-311. https://doi.org/10.5392/JKCA.2020.20.06.293

19. Internet Trend. Search engine [Internet]. Seoul: BizSpring Inc.; 2020 [cited 2020 August 6]. Available from: http://www.internettrend.co.kr/trendForward.tsp

20. Korea Centers for Disease Control. National health information portal [Internet]. Cheongju: Korea Centers for Disease Control; 2016 [cited 2020 August 6]. Available from: http://health.cdc.go.kr/health/Main.do

21. Textom. Big data solution textom manual [Internet]. Daegu: The IMC; 2019 [cited 2020 August 17]. Available from:

http://www.textom.co.kr/home/video/TEXTOM_v3_manual.pdf

22. Robertson SE. The probability ranking principle in IR. Journal of Documentation. 1977;33(4):294-304. https://doi.org/10.1108/eb026647

23. Mansouri V. Vocabulary Instuction: Software Flashcards vs. Word Clouds. Advances in Language and Literary Studies. 2015;6(1):41-45. https://doi.org/10.7575/aiac.alls.v.6n.1p.41
24. Beak YM. Text-mining using R. Paju: HanulMPlus; 2017. p. 13-235. 25. Brummelte S, Grunau RE, Synnes AR, Whitfield MF, PetrieThomas J. Declining cognitive development from 8 to 18 months in preterm children predicts persisting higher parenting stress. Early Human Development. 2011;87(4):273-280.

https://doi.org/10.1016/j.earlhumdev.2011.01.030

26. Pisoni C, Provenzi L, Moncecchi M, Caporali C, Naboni C, Stronati $\mathrm{M}$, et al. Early parenting intervention promotes 24-month psychomotor development in preterm children. Acta Paediatrica. 2020; 110(1):101-108. https://doi.org/10.1111/apa.15345

27. Cho ES, Kim SJ, Kwon MS, Kim EH, Jun EM, Lee S. The effect of kangaroo care in the neonatal intensive care unit on the physiological functions of preterm infants, maternal-infant attachment, and maternal stress. Journal of Pediatric Nursing. 2016;31(4): 430-438. https://doi.org/10.1016/j.pedn.2016.02.007

28. Kim MA, Kim SH, Cho H. Effects of tactile stimulation by fathers on physiological responses and paternal attachment in infants in the NICU: A pilot study. Jorunal of Child Health Care. 2017;21(1): 36-45. https://doi.org/10.1177/1367493516666729

29. Lee MS, Koh YH. Analysis of parental capacity to seek ways of child care support. Journal of Early Childhood Education and Educare Welfare. 2016;20(4):435-456.

30. Jeong BM, Kim NH. Parent educational experiences and needs of double income parents who have the first child in infancy. Korean Journal of Child Education. 2016;25(3):377-393.

https://doi.org/10.17643/KJCE.2016.25.3.20 